\title{
Ovals in $\mathbb{Z}_{2 p}^{2}$
}

\section{Zofia Stȩpień(i)}

\begin{abstract}
By an oval in $\mathbb{Z}_{2 p}^{2}, p$ odd prime, we mean a set of $2 p+2$ points, such that no three of them are on a line. It is shown that ovals in $\mathbb{Z}_{2 p}^{2}$ only exist for $p=3,5$ and they are unique up to an isomorphism.
\end{abstract}

Mathematics Subject Classification. 05B99.

Keywords. Arc, Collinearity.

\section{Introduction}

A $k$-arc is a set of $k$ points, such that no three of them are collinear. Arcs are well studied in projective geometry, see, e.g., [1,9] and [2] for further references. Recall that any non-singular conic of $\operatorname{PG}(2, q)$ is a $(q+1)$-arc. If $K$ is any $k$ arc of $\mathrm{PG}(2, q)$ with $q$ odd, then $k \leq q+1$. A $(q+1)$-arc is called an oval. A famous theorem of Segre [9] tells us that for $q$ odd, every oval of $\mathrm{PG}(2, p)$ is a non-singular conic.

Many authors continue to study the classical problem in the context of Hjelmslev geometry, see, e.g., [3,4,8] and [6] for the definition of the abstract Hjelmslev plane. In this article, we consider similar questions in $\mathbb{Z}_{n}^{2}$. This modified problem is still interesting and it was investigated in $[5,7]$ and [10]. If $n=p$ is prime, the resulting space is just $\operatorname{AG}(2, p)$. The correspondence between projective and affine planes leads to the following facts: The maximum size of an arc in $\mathbb{Z}_{p}^{2}$ is $p+1$; every $(p+1)$-arc in $\mathbb{Z}_{p}^{2}$ is a non-singular conic.

The maximum size of a cap in $\mathbb{Z}_{2 p}^{2}, p$ odd prime, is $2 p+2$ (see Lemma 4.1 in [7] and Theorem 3.1 in [10]). We will call a $(2 p+2)$-arc in $\mathbb{Z}_{2 p}^{2}$ an oval in $\mathbb{Z}_{2 p}^{2}$. In this article, we completely solve the problem of the existence and the uniqueness of ovals in $\mathbb{Z}_{2 p}^{2}$.

We define a line in $\mathbb{Z}_{n}^{2}$ to be a subset of $\mathbb{Z}_{n}^{2}$ of the form:

$$
\{(x ; y): a x+b y+c=0\},
$$

where $\operatorname{gcd}(a ; b ; n)=1$. This line is denoted by $\ell_{[a ; b ; c]}$. 
By an automorphism of $\mathbb{Z}_{n}^{2}$, we mean a mapping from $\mathbb{Z}_{n}^{2}$ to $\mathbb{Z}_{n}^{2}$ which preserves arcs. Let $X, Y$ be arcs in $\mathbb{Z}_{n}^{2}$. We say that $X$ and $Y$ are isomorphic if there exists an automorphism of $\mathbb{Z}_{n}^{2}$ mapping $X$ to $Y$.

\section{The General Case}

Due to the Chinese remainder theorem, we have the following.

Lemma 2.1. Let $p$ be an odd prime, $\phi_{2}: \mathbb{Z}_{2 p}^{2} \rightarrow \mathbb{Z}_{2}^{2}, \phi_{p}: \mathbb{Z}_{2 p}^{2} \rightarrow \mathbb{Z}_{p}^{2}$ be reduction maps. Then, any three points $x, y, z \in \mathbb{Z}_{2 p}^{2}$ are collinear if and only if $\phi_{2}(x), \phi_{2}(y), \phi_{2}(z) \in \mathbb{Z}_{2}^{2}$ and $\phi_{p}(x), \phi_{p}(y), \phi_{p}(z) \in \mathbb{Z}_{p}^{2}$ are collinear.

From now on, we will use the notation of the previous Lemma.

Lemma 2.2. Let $p$ be an odd prime. Let $\sigma$ be an arbitrary permutation of $\mathbb{Z}_{2}^{2}$. Then, there exists an automorphism $F$ of $\mathbb{Z}_{2 p}^{2}$, such that $\phi_{2} \circ F=\sigma \circ \phi_{2}$ and $\phi_{p} \circ F=I d \circ \phi_{p}$.

Proof. Let $F_{A}$ and $F_{B}$ be linear transformations determined by matrices $A=$ $\left(\begin{array}{ll}1 & 0 \\ p & 1\end{array}\right)$ and $B=\left(\begin{array}{cc}p+1 & p \\ p & p+1\end{array}\right)$, respectively. Denote by $t_{[0, p]}$ the translation by a vector $[0, p]$. Note that the group of permutations of the set $\mathbb{Z}_{2}^{2}$ is generated by transpositions $\sigma_{1}=((0,0),(0,1)), \sigma_{2}=((0,1),(1,0)), \sigma_{3}=((1,0),(1,1))$.

One can verify by a straightforward calculation that $\phi_{2} \circ F_{A} \circ t_{[0, p]}=$ $\sigma_{1} \circ \phi_{2}, \phi_{2} \circ F_{B}=\sigma_{2} \circ \phi_{2}$ and $\phi_{2} \circ F_{A}=\sigma_{3} \circ \phi_{2}$.

Lemma 2.3. Let $X$ be an oval in $\mathbb{Z}_{2 p}$. Then, the following holds.

(1) $\left|\phi_{2}(X)\right|=4$.

(2) $\left|\phi_{2}^{-1}(a) \cap X\right|=\frac{p+1}{2}$ for every $a \in \mathbb{Z}_{2}^{2}$.

(3) $\left|\phi_{p}(X)\right|=2 p+2$.

Proof. To prove (1), suppose that $\left|\phi_{2}(X)\right|<4$. Note that in this case, there exists a line $l$ in $\mathbb{Z}_{2}^{2}$, such that $\left|\phi_{2}^{-1}(l) \cap X\right|>p+1$. As a consequence of Lemma 2.1 together with the fact that the maximum size of an arc in $\mathbb{Z}_{p}$ is $p+1$, we obtain a contradiction with the assumption that $X$ is an arc. (2) follows immediately from (1) and Lemma 2.2. To prove (3), suppose, to the contrary, that $\phi_{p}(a)=\phi_{p}(b)$ for some $a, b \in X$. By (2), there exists a $c \in X$, such that $\phi_{2}(a)=\phi_{2}(c)$, so there exists a $c$, such that $\phi_{2}(a), \phi_{2}(b)$ and $\phi_{2}(c)$ are collinear. Moreover, it is clear that $\phi_{p}(a), \phi_{p}(b)$, and $\phi_{p}(c)$ are collinear. Therefore, $a, b, c$ is a collinear triple by Lemma 2.1 , a contradiction.

Theorem 2.4. There is no oval in $\mathbb{Z}_{2 p}$ for $p \geq 7$.

Proof. It is shown in [10] by computer calculations that the maximum size of an arc in $\mathbb{Z}_{14}$ is 12 . Assume, to the contrary, that there is an oval $X$ in $\mathbb{Z}_{2 p}$, where $p>7$ is an odd prime. By Lemma $2.3(1)$, we have $\phi_{2}(X)=\mathbb{Z}_{2}^{2}$. Let $a \in \mathbb{Z}_{2}^{2}$. By Lemma $2.3(2),(3),\left|\phi_{p}\left(\phi_{2}^{-1}(a) \cap X\right)\right|>4$. Denote by $\ell$ a line through $a$ in $\mathbb{Z}_{2}^{2}$. By Lemma $2.3(2)$ and (3) and Lemma 2.1, $\phi_{p}\left(\phi_{2}^{-1}(\ell) \cap X\right)$ is a $(p+1)$-arc in $\mathbb{Z}_{p}^{2}$, i.e., $\phi_{p}\left(\phi_{2}^{-1}(\ell) \cap X\right)$ is a non-singular conic. Note that there are exactly three distinct lines through $a$ in $\mathbb{Z}_{2}^{2}$, Therefore, we have three 


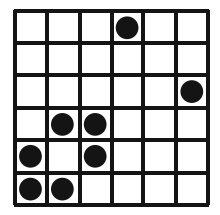

Figure 1. An oval in $\mathbb{Z}_{6}^{2}$

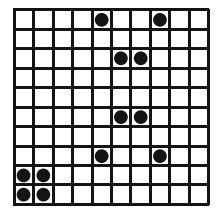

Figure 2. An oval in $\mathbb{Z}_{10}^{2}$

distinct non-singular conics through $\phi_{p}\left(\phi_{2}^{-1}(a) \cap X\right)$ in $Z_{p}$. However, this is a contradiction to the fact that there exists a unique non-singular conic through five points, no three collinear.

\section{The Cases $p=3,5$}

The existence of ovals in $\mathbb{Z}_{6}^{2}$ and $\mathbb{Z}_{10}^{2}$ was proved in [10], where appropriate examples were given, see also Figs. 1 and 2. Therefore, it remains to show the uniqueness.

First, we introduce some notation. Let $X \subset \mathbb{Z}_{2 p}^{2}$ be an oval. For $(i, j) \in$ $\mathbb{Z}_{2}^{2}$, let $X_{i j}=\phi_{p}\left(\phi_{2}^{-1}(i, j) \cap X\right)$. A $d$-secant of $K$ is a line $l$, such that $|l \cap K|=d$. Denote by $\mathcal{S}_{K}^{d}$ the set of $d$-secants of $K$.

The following Lemma is an immediate consequence of Lemma 2.1.

Lemma 3.1. Let $X$ be an oval in $\mathbb{Z}_{2 p}^{2}, a, b \in X$. If $\phi_{2}(a)=\phi_{2}(b)$, then the line through $\phi_{p}(a)$ and $\phi_{p}(b)$ is a 2 -secant of $\phi_{p}(X)$.

Theorem 3.2. An oval in $\mathbb{Z}_{6}$ is unique up to an isomorphism.

Proof. Let $Y$ be an oval in $\mathbb{Z}_{6}$ given in Fig. 1. This means that:

$$
Y=\{(0,0),(0,1),(1,0),(1,2),(2,1),(2,2),(3,5),(5,3)\} .
$$

Then, $\left|\phi_{3}(Y)\right|=8, Y_{00}=\{(0,0),(2,2)\}, \quad Y_{01}=\{(0,1),(2,1)\}$, $Y_{10}=\{(1,0),(1,2)\}, Y_{11}=\{(0,2),(2,0)\}$. Let $X$ be another oval in $\mathbb{Z}_{6}^{2}$. We will show that $X$ is isomorphic to $Y$. By Lemma $2.3(3),\left|\phi_{3}(X)\right|=8$. Let $(i, j) \in \mathbb{Z}_{2}^{2}$. Using Lemma $2.3(2)$ and (3), we obtain $\left|X_{i j}\right|=2$. From Lemma 3.1, it follows that $X_{i j}$ is a 2-secant of $\phi_{3}(X)$. Since the translations are automorphisms of $\mathbb{Z}_{6}^{2}$, we can assume that $(1,1) \notin \phi_{3}(X)$. This means that the points of $X_{i j}$ lie on one of the 4 lines through $(1,1)$. Thus, there exists $\left(i^{\prime}, j^{\prime}\right) \in \mathbb{Z}_{2}^{2}$, such that $X_{i j}=Y_{i^{\prime} j^{\prime}}$. Consequently, we obtain the permutation $\sigma$ of $\mathbb{Z}_{2}^{2}$ given by $\sigma(i, j)=\left(i^{\prime}, j^{\prime}\right)$. The proof is completed by Lemma 2.2 . 
The proof of Theorem 3.6 is straightforward but tedious. Therefore, we collect some useful Lemmas.

Lemma 3.3. Let $X, Y$ be ovals in $\mathbb{Z}_{10}^{2}$. If $\phi_{5}(X)$ and $\phi_{5}(Y)$ are isomorphic, then $X$ and $Y$ are isomorphic.

Proof. First, we will show that for every automorphism $f$ of $Z_{5}^{2}$, there exists an automorphism $F$ of $Z_{10}^{2}$, such that $\phi_{5} \circ F=f \circ \phi_{5}$. If $f$ is a translation by a vector, then we may take $F=f$. Obviously, $F$ satisfies the required condition. Let $f$ be an automorphism arising from multiplication by $A \in G L\left(2, \mathbb{Z}_{5}\right)$. It can be verified that at least one of $A: A+\left(\begin{array}{ll}5 & 0 \\ 0 & 0\end{array}\right), A+\left(\begin{array}{ll}0 & 5 \\ 0 & 0\end{array}\right), A+\left(\begin{array}{ll}0 & 0 \\ 5 & 0\end{array}\right), A+\left(\begin{array}{ll}0 & 0 \\ 0 & 5\end{array}\right)$, $A+\left(\begin{array}{ll}5 & 0 \\ 0 & 5\end{array}\right)$ is irreducible in $\mathbb{Z}_{10}$, call it $\tilde{A}$. Then, $F$ given by multiplication by $\tilde{A}$ satisfies the required condition.

Next, we claim that $f\left(X_{i j}\right)=Y_{i^{\prime} j^{\prime}}$. Suppose, to the contrary, that $f\left(X_{i j}\right)$ has a nonempty intersection with at least two of $Y_{00}, Y_{01}, Y_{10}, Y_{11}$. Recall that by Lemma 2.1, there exist six conics $C_{i}, i=1, \ldots, 6$, in $Y$ corresponding to six lines in $\mathbb{Z}_{2}^{2}$. Consider a conic $C$ containing $X_{i j}$. Then, $f(C)$ is the conic different from $C_{i}, i=1, \ldots, 6$. Moreover, there exists $C_{i}$ for some $i$, such that $\left|C \cup C_{i}\right| \geq 5$. However, this contradicts the fact that there exists a unique non-singular conic through five points, no three collinear.

Finally, consider the permutation $\sigma$ of $\mathbb{Z}_{2}^{2}$ given by $\sigma(i, j)=\left(i^{\prime}, j^{\prime}\right)$. Denote by $F_{1}$ an automorphism described in Lemma 2.2. Then, the composition of $F$ and $F_{1}$ maps $X$ onto $Y$.

Lemma 3.4. Let $X \subset \mathbb{Z}_{10}^{2}$ be an oval, $F(x, y)=(-x, y)$. If $F\left(X_{00}\right)=X_{01}$ and $F\left(X_{10}\right)=X_{00}$, then $\ell_{[1,0,0]} \notin \mathcal{S}_{X_{10}}^{2} \cup \mathcal{S}_{X_{11}}^{2}$.

Proof. Since $X_{10}$ is an arc, we have $\ell_{[1,0,0]} \in \mathcal{S}_{X_{10}}^{k}$ for $k=0,1,2$. Suppose, to the contrary, that $\ell_{[1,0,0]} \in \mathcal{S}_{X_{10}}^{2}$, i.e., $\left|X_{10} \cap \ell_{[1,0,0]}\right|=2$. Obviously $\mid X_{10} \cap$ $F\left(X_{10}\right) \mid=2$ and $X_{10} \neq F\left(X_{10}\right)$. We obtain ovals, i.e., non-singular conics, $C_{1}=X_{00} \cup X_{10}$ and $C_{2}=F\left(X_{01} \cup X_{10}\right)=X_{00} \cup F\left(X_{10}\right)$ with $\left|C_{1} \cap C_{2}\right|=5$. However, this contradicts the fact that there exists a unique non-singular conic through five points, no three collinear. Similarly, $\ell_{[1,0,0]} \notin \mathcal{S}_{X_{11}}^{k}$.

Lemma 3.5. Let $X \subset \mathbb{Z}_{10}^{2}$ be an oval. Then, the following holds:

(1) $\mathcal{S}_{X_{10}}^{2} \cup \mathcal{S}_{X_{11}}^{2} \subset \mathcal{S}_{X_{00} \cup X_{01}}^{0}$;

(2) $X_{i j}=\left\{l_{1} \cap l_{2}, l_{1} \cap l_{3}, l_{2} \cap l_{3}\right\}$, where $\mathcal{S}_{X_{i j}}^{2}=\left\{l_{1}, l_{2}, l_{3}\right\}$.

Proof. (1) is a direct consequence of Lemma 2.1. (2) follows from the definition of $\mathcal{S}_{X_{i j}}^{2}$ together with the fact that both $X_{i j}$ and $\mathcal{S}_{X_{i j}}^{2}$ have size 3 .

We are now ready for the proof of Theorem 3.6.

Theorem 3.6. An oval in $\mathbb{Z}_{10}$ is unique up to an isomorphism.

Proof. Let $Y$ be an oval in $\mathbb{Z}_{10}$ given in Fig. 2. This means that:

$Y=\{(0,0),(1,0),(0,1),(1,1),(4,2),(7,2),(5,4),(6,4),(5,7),(6,7),(4,9),(7,9)\}$ 
Let $X$ be another arc of size 12 in $\mathbb{Z}_{10}^{2}$. We will show that $X$ is isomorphic to $Y$. Recall that $X_{00} \cup X_{01}$ is a non-singular conic $C$ in $\mathbb{Z}_{5}^{2}$. Thus, $C$ can be transformed by some automorphism of $\mathbb{Z}_{5}^{2}$ to $C_{1}=\left\{(x, y): x^{2}+2 y^{2}=1\right\}$. By Lemma 3.3, we can write $X_{00} \cup X_{01}=\{(1,0),(2,1),(2,4),(4,0),(3,1),(3,4)\}$.

It is straightforward to check that in this case, we have:

$$
\mathcal{S}_{X_{00} \cup X_{01}}^{0}=\left\{\ell_{[1 ; 0 ; 0]}, \ell_{[1 ; 1 ; 0]}, \ell_{[4 ; 1 ; 0]}, \ell_{[0 ; 1 ; 2]}, \ell_{[0 ; 1 ; 3]}, \ell_{[3 ; 1 ; 1]}, \ell_{[3 ; 1 ; 4]}, \ell_{[2 ; 1 ; 1]}, \ell_{[2 ; 1 ; 4]}\right\}
$$

We need to split $X_{00} \cup X_{01}$ into two subsets $X_{00}, X_{01}$. By Lemma 2.2, we may assume that $(1,0) \in X_{00}$. There are ten cases for $X_{00}$. To reduce the number of cases, we use Lemma 3.3 and the automorphism $G$ of $\mathbb{Z}_{5}^{2}$ given by $G(x, y)=(3 x+3 y, x+3 y)$. A straightforward computation shows that it remains to consider the following three cases: (1) $X_{00}=\{(1,0),(3,1),(3,4)\}$, (2) $X_{00}=\{(1,0),(2,1),(2,4)\},(3) X_{00}=\{(1,0),(2,1),(3,4)\}$.

We shall consider the cases (1) and (2) separately, and then, we will show that cases 1 and 3 give isomorphic ovals.

Case $1 X_{00}=\{(1,0),(3,1),(3,4)\}$. Then, $X_{01}=\{(4,0),(2,1),(2,4)\}$. It is straightforward to check that $\mathcal{S}_{X_{00}}^{2}=\left\{\ell_{[2 ; 1 ; 3]}, \ell_{[3 ; 1 ; 2]}, \ell_{[1 ; 0 ; 2]}\right\}$ and $\mathcal{S}_{X_{01}}^{2}=$ $\left\{\ell_{[3 ; 1 ; 3]}, \ell_{[2 ; 1 ; 2]}, \ell_{[1 ; 0 ; 3]}\right\}$. Let $\left\langle\mathcal{S}_{X_{i j}}^{2}\right\rangle=\left\{p \in \ell \mid \ell \in \mathcal{S}_{X_{i j}}^{2}\right\}$. Denote by $\overline{\left\langle\mathcal{S}_{X_{i j}}^{2}\right\rangle}$ the complement of $\left\langle\mathcal{S}_{X_{i j}}^{2}\right\rangle$. Next, one can verify that $\overline{\left\langle\mathcal{S}_{X_{00}}^{2}\right\rangle} \cap \overline{\left\langle\mathcal{S}_{X_{01}}^{2}\right\rangle}=$ $\{(0,0),(0,1),(0,4),(1,2),(1,3),(4,2),(4,3)\}$. It follows from Lemma 3.1 that $X_{10} \cup X_{11} \subset \overline{\left\langle\mathcal{S}_{X_{00}}^{2}\right\rangle} \cap \overline{\left\langle\mathcal{S}_{X_{01}}^{2}\right\rangle}$. Furthermore, note that $(0,0)$ is only one point of $\ell_{[4 ; 1 ; 0]}$ in $\overline{\left\langle\mathcal{S}_{X_{00}}^{2}\right\rangle} \cap \overline{\left\langle\mathcal{S}_{X_{01}}^{2}\right\rangle}$. Thus, $\ell_{[4 ; 1 ; 0]} \notin \mathcal{S}_{X_{10}}^{2} \cup \mathcal{S}_{X_{11}}^{2}$. By the same argument, $\ell_{[1 ; 1 ; 0]} \notin \mathcal{S}_{X_{10}}^{2} \cup \mathcal{S}_{X_{11}}^{2}$. Lemma 3.4 implies that $\ell_{[1 ; 0 ; 0]} \notin \mathcal{S}_{X_{10}}^{2} \cup$ $\mathcal{S}_{X_{11}}^{2}$. The above considerations together with Lemma 3.5 (1) and Eq. (3.0.1) imply that $\mathcal{S}_{X_{10}}^{2} \cup \mathcal{S}_{X_{11}}^{2}=\left\{\ell_{[0 ; 1 ; 2]}, \ell_{[0 ; 1 ; 3]}, \ell_{[3 ; 1 ; 1]}, \ell_{[3 ; 1 ; 4]}, \ell_{[2 ; 1 ; 1]}, \ell_{[2 ; 1 ; 4]}\right\}$. (We use the fact that $\left|\mathcal{S}_{X_{10}}^{2} \cup \mathcal{S}_{X_{11}}^{2}\right|=6$.) Lemma $3.5(2)$ and simple calculations show that $(0,0) \notin X_{10} \cup X_{11}$. Since $\left|X_{10} \cup X_{11}\right|=6, X_{10} \cup X_{11}=$ $\{(0,1),(0,4),(1,2),(1,3),(4,2),(4,3)\}$. Now, we need to split $X_{10} \cup X_{11}$ into two subsets $X_{10}, X_{11}$. By Lemma 2.2 , we may assume that $(0,1) \in X_{10}$. It can be verified that $\{(0,1)\}=\ell_{[3 ; 1 ; 4]} \cap \ell_{[2 ; 1 ; 4]}$. By Lemma $3.5(2), \ell_{[3 ; 1 ; 4]}, \ell_{[2 ; 1 ; 4]} \in$ $\mathcal{S}_{X_{10}}^{2}$. One can verify that $\ell_{[3 ; 1 ; 4]} \cap\left(X_{10} \cup X_{11}\right)=\{(0,1),(1,3)\}$ and $\ell_{[2 ; 1 ; 4]} \cap$ $\left(X_{10} \cup X_{11}\right)=\{(0,1),(4,3)\}$. Hence, $X_{10}=\{(0,1),(1,3),(4,3)\}$, and so, $X_{11}=\{(0,4),(1,2),(4,2)\}$

Thus:

$$
X=\{(6,0),(8,6),(8,4),(4,5),(2,1),(2,9),(5,6),(1,8),(9,8),(5,9),(1,7),(9,7)\} .
$$

Finally, one can check that $H(X)=Y$, where $H(x, y)=(3 x+7 y+8,4 x+$ $9 y+3)$.

Case $2 X_{00}=\{(1,0),(2,1),(2,4)\}$. Then, $X_{01}=\{(4,0),(3,1),(3,4)\}$. In this case, $\mathcal{S}_{X_{00}}^{2}=\left\{\ell_{[4 ; 1 ; 1]}, \ell_{[1 ; 0 ; 3]}, \ell_{[1 ; 1 ; 4]}\right\}$ and $\mathcal{S}_{X_{01}}^{2}=\left\{\ell_{[4 ; 1 ; 4]}, \ell_{[1 ; 0 ; 2]}, \ell_{[1 ; 1 ; 1]}\right\}$. With the notation of the above case, it can be verified that:

$$
\overline{\left\langle\mathcal{S}_{X_{00}}^{2}\right\rangle} \cap \overline{\left\langle\mathcal{S}_{X_{01}}^{2}\right\rangle}=\{(0,0),(0,2),(0,3),(1,1),(1,4),(4,1),(4,4)\} .
$$


Hence, $X_{10} \cup X_{11} \subset\{(0,0),(0,2),(0,3),(1,1),(1,4),(4,1),(4,4)\}$, see Lemma 3.1. By the same arguments as in the previous case, $\ell_{[1 ; 0 ; 0]}, \ell_{[0 ; 1 ; 3]}, \ell_{[0 ; 1 ; 2]}$ $\notin \mathcal{S}_{X_{10}}^{2} \cup \mathcal{S}_{X_{11}}^{2}$ and, therefore, $\mathcal{S}_{X_{10}}^{2} \cup \mathcal{S}_{X_{11}}^{2}=\left\{\ell_{[1 ; 1 ; 0]}, \ell_{[4 ; 1 ; 0]}, \ell_{[3 ; 1 ; 1]}, \ell_{[3 ; 1 ; 4]}, \ell_{[2 ; 1 ; 1]}\right.$, $\left.\ell_{[2 ; 1 ; 4]}\right\}$. It can be verified that $\ell_{[3 ; 1 ; 4]} \cap \ell_{[2 ; 1 ; 4]}=\{(0,1)\}$. However, $(0,1) \notin$ $X_{10} \cup X_{11}$. This contradicts Lemma $3.5(2)$. Hence, this case does not occur.

Finally, we will show that cases 1 and 3 give isomorphic ovals. For $(i, j) \in$ $\mathbb{Z}_{2}^{2}$ and $k=1,3$, denote by $X_{i j}^{k}$ the set $X_{i j}$ from Case $k$. With this notation, $X_{00}^{3}=\{(1,0),(2,1),(3,4)\}$ and $X_{01}^{3}=\{(4,0),(2,4),(3,1)\}$. Consider $f$ given by $f(x, y)=(3 x+2 y, 3 x+3 y+2)$. One easily verifies that $f\left(X_{11}^{1}\right)=X_{00}^{3}$ and $f\left(X_{01}^{1}\right)=X_{01}^{3}$. The result now follows from Lemma 3.3. The proof is complete.

Open Access. This article is licensed under a Creative Commons Attribution 4.0 International License, which permits use, sharing, adaptation, distribution and reproduction in any medium or format, as long as you give appropriate credit to the original author(s) and the source, provide a link to the Creative Commons licence, and indicate if changes were made. The images or other third party material in this article are included in the article's Creative Commons licence, unless indicated otherwise in a credit line to the material. If material is not included in the article's Creative Commons licence and your intended use is not permitted by statutory regulation or exceeds the permitted use, you will need to obtain permission directly from the copyright holder. To view a copy of this licence, visit http://creativecommons. org/licenses/by/4.0/.

Publisher's Note Springer Nature remains neutral with regard to jurisdictional claims in published maps and institutional affiliations.

\section{References}

[1] R. C. Bose, Mathematical Theory of the Symmetrical Factorial Design Sankhya: The Indian Journal of Statistics (1933-1960) Vol. 8, No. 2 (Mar., 1947), 107-166

[2] J.W.P. Hirschfeld, Projective Geometries over Finite Fields, Clarendon Press, Oxford, 1998

[3] T. Honold, I. Landjev, On arcs in projective Hjelmslev planes, Discrete Math. 231 (2001), no. 1-3, 265-278

[4] T. Honold, I. Landjev, On maximal arcs in projective Hjelmslev planes over chain rings of even characteristic, Finite Fields Appl. 11 (2005), no. 2, 292-304

[5] J. Huizenga, The minimum size of complete caps in $(\mathbb{Z} / n \mathbb{Z})^{2}$, Electron. J. Combin. 13 (2006), no. 1, Research Paper 58, 19 pp.

[6] E. Kleinfeld, Finite Hjelmslev planes, Illinois J. Math. 3 (1959), 403-407

[7] S. Kurz, Caps in $\mathbb{Z}_{n}^{2}$, Serdica J. Computing 3 (2009), 159-178 
[8] M. Kiermaier, M. Koch, S. Kurz, 2-arcs of maximal size in the affine and the projective Hjelmslev plane over $Z_{25}$, Adv. Math. Commun. 5 (2011), no. 2, 287301.

[9] B. Segre, Ovals in a finite projective plane, Canad. J. Math. 7, (1955), 414-416

[10] Z. Stȩpień, L. Szymaszkiewicz, Arcs in $\mathbb{Z}_{2 p}^{2}$, Journal of Combinatorial Optimization (2018), Volume 35, no. 2, 341-349.

\section{Zofia Stȩpień}

School of Mathematics

West Pomeranian University of Technology Szczecin

al. Piastów 48/49

70-310 Szczecin

Poland

e-mail: stepien@zut.edu.pl

Received: 11 December 2019.

Accepted: 20 June 2020. 